\title{
Multispectral Optoacoustic Tomography of Benign and Malignant Thyroid Disorders: A Pilot Study
}

\author{
Wolfgang Roll*1, Niklas A. Markwardt*2,3, Max Masthoff ${ }^{4}$, Anne Helfen ${ }^{4}$, Jing Claussen ${ }^{5}$, Michel Eisenblätter ${ }^{4,6}$, \\ Alexa Hasenbach ${ }^{7}$, Sven Hermann ${ }^{7}$, Angelos Karlas ${ }^{2,3}$, Moritz Wildgruber ${ }^{4,8}$, Vasilis Ntziachristos*2,3, \\ and Michael Schäfers*1,7,8
}

\begin{abstract}
${ }^{1}$ Department of Nuclear Medicine, University Hospital Münster, Münster, Germany; ${ }^{2}$ Institute of Biological and Medical Imaging, Helmholtz Zentrum München, München, Germany; ${ }^{3}$ Chair of Biological Imaging and TranslaTUM, Technische Universität München, München, Germany; ${ }^{4}$ Institute of Clinical Radiology, University Hospital Münster, Münster, Germany; ${ }^{5}$ iThera Medical, Munich, Germany; ${ }^{6}$ Division of Imaging Sciences and Biomedical Engineering, King's College London, London, United Kingdom; ${ }^{7}$ European Institute for Molecular Imaging, University of Münster, Münster, Germany; and ${ }^{8}$ Cells in Motion (CiM) Cluster of Excellence, University of Münster, Münster, Germany
\end{abstract}

\begin{abstract}
This study aimed at evaluating hybrid multispectral optoacoustic tomography/ultrasound for imaging of thyroid disorders, including Graves' disease and thyroid nodules. Methods: The functional biomarkers and tissue parameters deoxygenated hemoglobin, oxygenated hemoglobin, total hemoglobin, saturation of hemoglobin, fat content, and water content were analyzed in thyroid lobes affected by Graves' disease $(n=6)$, thyroid lobes with healthy tissue $(n=8)$, benign thyroid nodules $(n=13)$, and malignant thyroid nodules $(n=$ 3). Results: In Graves' disease, significantly higher deoxygenated hemoglobin $(3.18 \pm 0.52$ vs. $2.13 \pm 0.62 ; P=0.0055)$ and total hemoglobin (8.34 \pm 0.88 vs. $6.59 \pm 1.16 ; P=0.0084)$ and significantly lower fat content $(0.64 \pm 0.37$ vs. $1.69 \pm 1.25 ; P=0.0293)$ were found than in healthy controls. Malignant thyroid nodules showed significantly lower saturation of hemoglobin $(55.4 \% \pm$ $2.6 \%$ vs. $60.8 \% \pm 7.2 \% ; P=0.0393)$ and lower fat content $(0.62 \pm$ 0.19 vs. $1.46 \pm 0.87 ; P=0.1295)$ than benign nodules. Conclusion: This pilot study showed the applicability and the potential of hybrid multispectral optoacoustic tomography/ultrasound to semiquantitatively provide tissue characterization and functional parameters in thyroid disorders for improved noninvasive diagnostics of thyroid diseases.
\end{abstract}

Key Words: multispectral optoacoustic tomography (MSOT); Graves' disease; thyroid nodule; hemoglobin

J Nucl Med 2019; 60:1461-1466

DOI: 10.2967/jnumed.118.222174

$\mathbf{T}$ hyroid disorders, including autoimmune diseases and thyroid nodules, are common diseases worldwide. Graves' disease is one of the most frequent causes for hyperthyroidism, causing an antibody-mediated (autoimmune) inflammation of the thyroid gland $(1,2)$. Clinical evaluation and risk stratification include laboratory

Received Oct. 19, 2018; revision accepted Feb. 26, 2019

For correspondence or reprints contact: Wolfgang Roll, Department of Nuclear Medicine, University Hospital Münster, Albert-Schweitzer-Campus 1, 48149 Münster, Germany.

E-mail: wolfgang.roll@ukmuenster.de

${ }^{*}$ Contributed equally to this work.

Published online Mar. 8, 2019.

COPYRIGHT (c) 2019 by the Society of Nuclear Medicine and Molecular Imaging. testing of thyroid hormones (thyroid-stimulating hormone, free thyroxine, and free triiodothyronine), autoantibodies (measurement of thyrotropin receptor antibodies [TRAK, for Thyreotropin-RezeptorAntikörper]), and ultrasound/Doppler imaging (2). ${ }^{99 \mathrm{~m} T \mathrm{Tc}-\text { scintigra- }}$ phy is not recommended for routine monitoring of Graves' disease because of the related radiation exposure (2). Noninvasive and more specific functional monitoring of inflammatory activity as, for example, through multispectral optoacoustic tomography (MSOT) could improve the assessment of treatment response.

Thyroid nodules are detected in $19 \%-68 \%$ of individuals by high-resolution ultrasound $(3,4)$. Grading following the guidelines of the American Thyroid Association (5) or the criteria or modified criteria of the Thyroid Imaging Reporting and Documentation System $(6,7)$ helps to estimate the risk of malignancy based on ultrasound patterns and nodule sizes guiding fine-needle aspiration (FNA). Providing a low number of false-negative results, FNA is a powerful diagnostic tool for nonsurgical diagnosis (2). However, about $25 \%$ of thyroid nodules are classified as indeterminate and cannot be characterized as benign, malignant, or suggestive of malignancy with a high risk of cancer (8). Definite conclusions can be drawn only from histopathology after thyroidectomy or hemithyroidectomy $(9,10)$. Further evaluation and risk stratification imaging techniques such as the recently established elastography can be applied; however, these are operator-dependent and of highly variable performance (5). New noninvasive techniques such as MSOT, assessing functional tissue parameters, might provide new biomarkers, which would be highly desirable to further assess risk patterns for individual thyroid nodules without the need for invasive procedures.

MSOT is based on the detection of ultrasonic waves generated by thermoelastic expansion of tissue illuminated with ultrashort laser pulses (Fig. 1) (11). Distributions of different tissue parameters, for example, fat and water content or concentration of oxygenated hemoglobin $\left(\mathrm{HbO}_{2}\right)$ and deoxygenated hemoglobin (HbR), can be calculated because of their specific intrinsic absorption properties. Recently, first potential clinical applications for optoacoustic imaging in the fields of vascular imaging $(12,13)$, inflammatory bowel diseases (14), and oncology (15) have been reported. Regarding thyroid diseases, the initial results of optoacoustic imaging ex vivo $(16,17)$ recently triggered the first proof-of-concept imaging studies of healthy subjects (18) and patients with thyroid nodules in vivo (19). However, both studies applied only a single illumination wavelength, implying that the spectral signatures of 


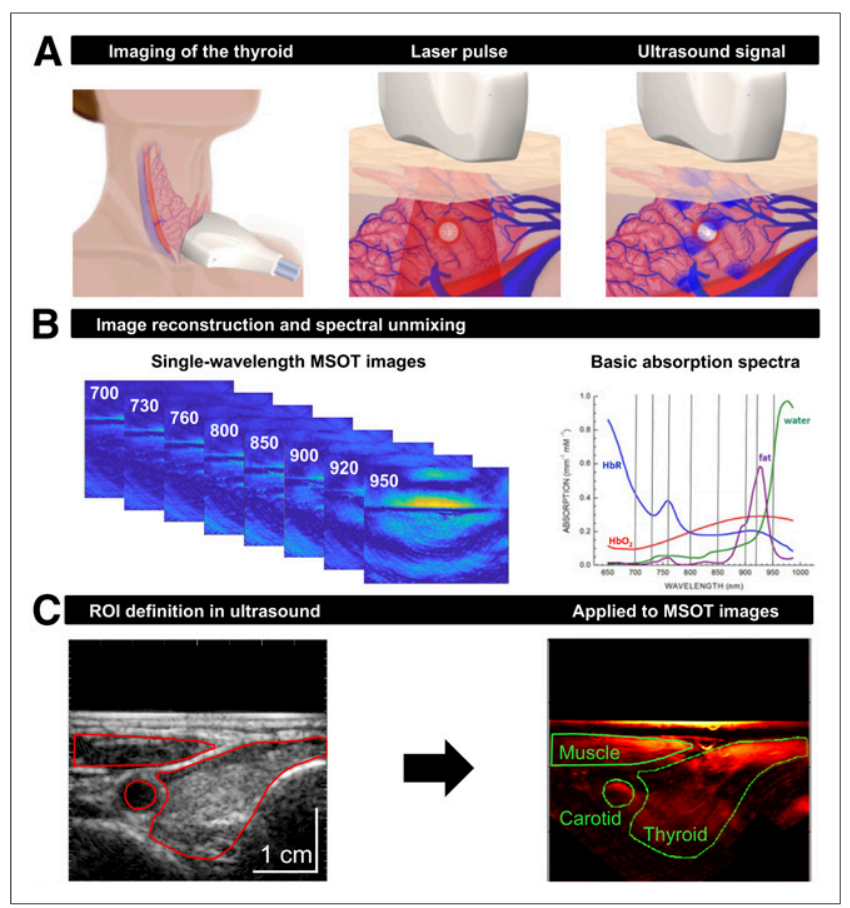

FIGURE 1. Principles of clinical MSOT of thyroid. (A) Scheme of examination of thyroid gland with handheld hybrid MSOT/ultrasound system (left). Patients with thyroid nodules, healthy individuals, and Graves' disease patients were scanned in a reproducible setup. Optoacoustic imaging is based on absorption of irradiated laser pulses within tissue (middle), followed by thermoelastic expansion and induction of ultrasound waves that can be detected with handheld detector (right). (B) In a first step, MSOT images are acquired for single wavelengths (left). Spectral unmixing, based on specific absorption spectra of different tissue constituents (right), allows assessment of functional parameters such as $\mathrm{HbR}, \mathrm{HbO}_{2}$, fat content, and water content. (C) Transversal ultrasound image of thyroid gland and surrounding tissue allows exact localization of anatomic structures (left). ROIs drawn on ultrasound images were transferred to coregistered pseudo colorcoded averaged MSOT images (here, $\mathrm{HbT}$ ) for visual and quantitative analysis (right).

different tissue absorbers could not be used to quantify functional parameters in thyroid disorders. In addition, the integration of MSOT with ultrasound imaging (a current major imaging modality in clinical diagnostics of the thyroid)—as implemented in the hybrid device used in this study-allows benefiting from both functional (through MSOT) and anatomic (through ultrasound) information coregistered in space and time.

The aim of this study was to evaluate the applicability and the potential of hybrid MSOT/ultrasound imaging in common disorders of the thyroid gland in patients compared with healthy controls. Functional tissue parameters provided by MSOT were used to assess inflammatory activity in Graves' disease and to further characterize thyroid nodules.

\section{MATERIALS AND METHODS}

\section{Patients and Study Design}

Eighteen patients (median age, $52 \mathrm{y}$; range, 21-82 y) were included, consisting of Graves' disease patients $(n=3)$ (Table 1$)$, healthy volunteers $(n=3)$, patients with only benign thyroid nodules $(n=9)$, and patients with a malignant thyroid nodule $(n=3)$.

For the comparison of Graves' disease with healthy thyroid tissue, 14 thyroid lobes (Graves' disease, $n=6$; healthy tissue, $n=8$ ) were included in this retrospective analysis. Both lobes (left and right) of the Graves' disease patients were affected by Graves' disease. The healthy-tissue lobes included 1 or 2 lobes per healthy volunteer $(n=4)$ and the contralateral, unaffected lobes of thyroid nodule patients $(n=4)$. The 2 other lobes of healthy volunteers were excluded because of the presence of small cystic lesions. Sixteen thyroid nodules were analyzed, consisting of 13 benign nodules (Supplemental Table 1; supplemental materials are available at http://jnm.snmjournals. org) and 3 malignant nodules (Table 2). All patients underwent a routine clinical thyroid evaluation in our nuclear medicine outpatient clinic. Graves' disease evaluation included medical history, laboratory testing of thyroid hormones (thyroid-stimulating hormone, free triiodothyronine, and free thyroxine), autoantibodies (TRAK), and ultrasound with Doppler imaging following international guidelines (2). Risk stratification of thyroid nodules included ultrasound imaging, ${ }^{99 \mathrm{~m}} \mathrm{Tc}$-pertechnetate scintigraphy, and, if recommended, FNA according to international guidelines $(5,6,20)$. The final diagnosis was based on histopathologic results after thyroidectomy in 4 benign and 3 malignant nodules (Supplemental Tables 1 and 2, respectively). FNA served as the gold standard for 4 nodules. Hyperfunctional nodules with high uptake on ${ }^{99 \mathrm{~m}} \mathrm{Tc}$-pertechnetate scintigraphy were regarded as benign $(n=5)$ and did not require FNA (5).

All patients and healthy subjects gave written informed consent before enrolment. This retrospective analysis was approved by the institutional review board (approval 2018-745-f-S).

\section{Technical Aspects of MSOT Imaging Device}

We used a hybrid clinical MSOT/ultrasound imaging system (MSOT Acuity Echo; iThera Medical) previously described in detail elsewhere $(15,21)$. Laser excitation pulses had a duration of $9 \mathrm{~ns}$ with a repetition rate of $25 \mathrm{~Hz}$. The pulse energy was attenuated to ensure adherence to the American National Standards Institute limits of maximum permissible exposure (energy density $<20 \mathrm{~mJ} / \mathrm{cm}^{2}$ ). The detector (256 transducer elements with a center frequency of $3 \mathrm{MHz}$; send/receive bandwidth of $56 \%$; optoacoustic resolution of $\sim 250 \mu \mathrm{m}$ ) had a $125^{\circ}$ angular coverage providing 2-dimensional cross-sectional

TABLE 1

Characteristics of Patients with Graves' Disease

\begin{tabular}{ccccccc}
\hline Study & Age at diagnosis $(\mathrm{y})$ & Thyroid volume $(\mathrm{mL})$ & Doppler & $\mathrm{fT}(\mathrm{pg} / \mathrm{mL})^{*}$ & TRAK (U/I) & Thyreostatics \\
\hline 1 & 45 & 21 & Hyperperfused & 4.9 & 12.3 & $10 \mathrm{mg}$ carbimazole \\
2 & 21 & 47 & Hyperperfused & 11.2 & $8,331.7$ & $60 \mathrm{mg}$ carbimazole \\
3 & 53 & 17 & Hyperperfused & 5.9 & 6.1 & $20 \mathrm{mg}$ thiamazole \\
\hline
\end{tabular}

${ }^{*}$ Range, 2.3-4.2.

fT3 $=$ free triiodothyronine. 
TABLE 2

Characteristics of Patients with Papillary Thyroid Carcinoma

\begin{tabular}{lcllc} 
& $\begin{array}{c}\text { Age at } \\
\text { Nodule }\end{array}$ & & \multicolumn{1}{c}{ TNM } \\
diagnosis $(\mathrm{y})$ & Localization & (UICC 2010) & Size $(\mathrm{cm})$ \\
\hline 1 & 24 & Right lobe & pT2 & 3.5 \\
2 & 21 & Left lobe & pT1b & 1.5 \\
3 & 66 & Left lobe & pT1a & 0.3
\end{tabular}

UICC $=$ Union for International Cancer Control.

images with a field of view of $40 \times 40 \mathrm{~mm}$ and a reconstructed pixel size of $100 \mu \mathrm{m}$. Multispectral data were acquired using 1 pulse per wavelength. Reflection CT-mode ultrasound images were generated as previously described (15).

\section{MSOT Image Acquisition}

MSOT imaging of the thyroid gland was conducted after routine clinical thyroid evaluation in our outpatient clinic on the same day. Scans were performed at room temperature under stable conditions with the patient lying supine in the same position as for routine ultrasound imaging. Image acquisition took about $5 \mathrm{~min}$, with the handheld probe (Fig. 1A) touching the skin most of the time. While acquiring images of different wavelengths, the probe was placed transversally and longitudinally centered on the largest extent of the thyroid lobe (Graves' disease, healthy lobe) or on the thyroid nodule. Breath-holding was required. The eyes of examiners and patients were protected with laser safety goggles (protection level, DIR LB3; wavelength range, 645-1,400 nm; visible light transmission, 40\%). Examiners were experienced in head and neck/thyroid ultrasound and clinical optoacoustic imaging.

MSOT images were acquired at 8 wavelengths ranging from 700 to $950 \mathrm{~nm}(700,730,760,800,850,900,920$, and $950 \mathrm{~nm})$.

\section{Image Reconstruction and Data Analysis}

MSOT images were reconstructed using a model-based algorithm with Tikhonov regularization and nonnegativity constraint (22) after band-pass filtering (Chebyshev) between $90 \mathrm{kHz}$ and $6 \mathrm{MHz}$ and deconvolution with the electrical impulse response of the transducer. A single, effective speed of sound of $1,510 \mathrm{~m} / \mathrm{s}$ was assumed for tissue and coupling medium (heavy water). This reconstruction method was used to ensure optimal coregistration of MSOT and ultrasound images.

Individual contributions of the absorbers $\mathrm{HbO}_{2}, \mathrm{HbR}$, fat, and water were recovered from the acquired data on the basis of their spectral absorption characteristics by linear unmixing. For the unmixing of $\mathrm{HbO}_{2}$ and $\mathrm{HbR}$, only the wavelengths $700,730,760$, 800 , and $850 \mathrm{~nm}$ were used; for fat and water all wavelengths were used (i.e., also $900,920$, and $950 \mathrm{~nm})$. Subsequently, total hemoglobin $\left(\mathrm{HbT}=\mathrm{HbO}_{2}+\mathrm{HbR}\right)$ and oxygen saturation $\left(\mathrm{sO}_{2}=\mathrm{HbO}_{2} / \mathrm{HbT}\right)$ were calculated.

To increase the signal-to-noise ratio, 2 to 3 frame stacks representing different time intervals in the image sequence of a scan were evaluated and averaged. The time intervals were chosen to exhibit no significant detector or patient movement. Each frame stack comprised 5 sequential multispectral frames, each consisting of 8 single-wavelength slices (Fig. 1B).

Regions of interest (ROIs) were drawn on ultrasound images and transferred to the corresponding coregistered MSOT images. The pixelwise calculated unmixed absorber concentrations were averaged in the ROIs. In healthy tissue lobes and Graves' disease lobes, the ROIs were placed surrounding the whole lobe visible in the ultrasound image (Fig. 1C). In patients with thyroid nodules, the ROIs were placed surrounding the entire nodule (Figs. 2A and 2B) by a nuclear medicine specialist.

Statistical analysis was performed with MATLAB (version R2017b; The MathWorks, Inc.). Grouped data were compared using the following scheme: if both groups were normally distributed, a Student $t$ test (for equal variances) or a Welch test (for different variances) was used; if at least one of the groups was not normally distributed, a WilcoxonMann-Whitney test was applied. Results are indicated in the text as mean $\pm 1 \mathrm{SD}$ and 
visualized in the figures as single points (Graves' disease/healthy tissue, single lobes; nodules, single nodules), with additional boxes indicating $\pm 1 \mathrm{SD}$ and additional lines representing the respective means. $P$ values of less than 0.05 were considered significant.

\section{RESULTS}

Hybrid MSOT/ultrasound, aside from requiring laser safety goggles, was as easily applicable to patients with thyroid diseases and controls as ultrasound alone, allowing for noninvasive and semiquantitative analysis of functional parameters integrated with anatomic information. These parameters were also sufficiently reproducible: the relative SD referring to the 2- to 3-frame stacks used for the evaluation of each scan, averaged over all scans, remained below $10 \%\left(\mathrm{HbR}, 4.9 \% ; \mathrm{HbO}_{2}, 9.1 \%\right.$; $\mathrm{HbT}, 5.9 \% ; \mathrm{sO}_{2}$, $4.4 \%$; water, $6.4 \%$ ), or at least below $20 \%$ in the case of fat (16.1\%). Similarly, contralateral lobes in healthy volunteers and Graves' disease patients showed similar results (as expected). Their deviations from the respective patient averages were reasonably small: $5.0 \%, 7.8 \%, 5.3 \%, 2.9 \%, 26.4 \%$, and $9.4 \%$ for $\mathrm{HbR}$, $\mathrm{HbO}_{2}, \mathrm{HbT}, \mathrm{sO}_{2}$, fat, and water, respectively.

\section{Graves' Disease}

In Figure 3A, HbR, HbT, and fat images of healthy tissue and tissue affected by Graves' disease are exemplarily shown for 1 lobe of each group, highlighting the significant differences presented in Figure 3B. In thyroid lobes affected by Graves' disease, $\operatorname{HbR}(3.18 \pm 0.52$ vs. $2.13 \pm 0.62 ; P=0.0055)$ and $\mathrm{HbT}(8.34 \pm$ 0.88 vs. $6.59 \pm 1.16 ; P=0.0084)$ were significantly higher than in control tissue, whereas the fat content $(0.64 \pm 0.37$ vs. $1.69 \pm$ $1.25 ; P=0.0293$ ) was significantly lower. $\mathrm{HbO}_{2}, \mathrm{sO}_{2}$, and water content did not differ significantly. Additionally, there were no significant differences in any of the 6 parameters between contralateral, unaffected lobes of thyroid nodule patients and lobes of healthy volunteers (both classified as healthy tissue).

\section{Thyroid Nodules}

The upper panels of Figure 2 show an example capsulated benign nodule, which is well seen in MSOT. The dark (low-echo) rim in the raw ultrasound image (Fig. 2A) delineates the nodule. Drawing an ROI just along this rim (Fig. 2B) and transferring it to the MSOT image ( $\mathrm{sO}_{2}$ image weighted with the HbT signal) helps identify the nodule as the region with comparably high $\mathrm{sO}_{2}$ (Fig. 2C).

The results of MSOT parameters in benign and malignant nodules are shown in Figure 2D. A significant difference can be observed for $\mathrm{sO}_{2}$, which was lower in malignant nodules $(55.4 \%$ $\pm 2.6 \%)$ than in benign nodules $(60.8 \% \pm 7.2 \%)(P=0.0393)$. This difference would be even more striking without an outlier in the benign group with a remarkably low $\mathrm{sO}_{2}$ of about $40 \%$ (indicated by an arrow in Fig. 2D). This nodule was located just below a large blood vessel, which probably distorted the measured values. Furthermore, malignant nodules showed lower fat content $(0.62 \pm 0.19)$ than benign nodules (1.46 \pm 0.87 , but the difference did not reach statistical significance in the small patient sample of this pilot study $(P=0.1295)$. $\mathrm{HbO}_{2}$, $\mathrm{HbT}$, and water content did not differ significantly.
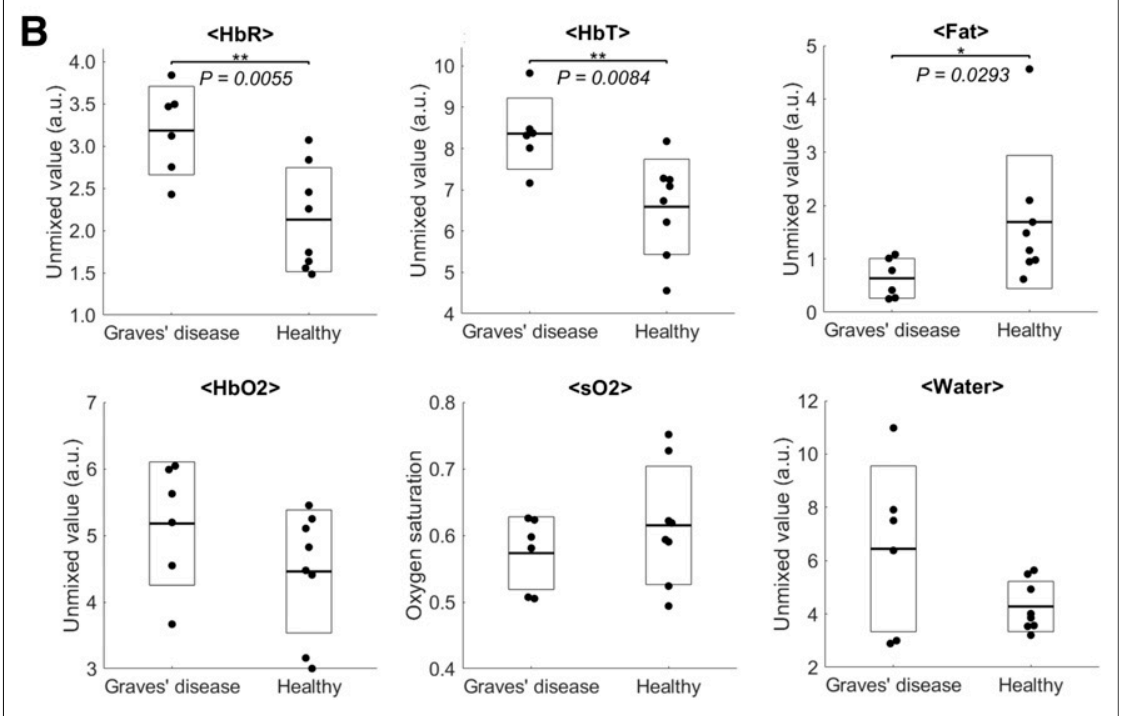

FIGURE 3. MSOT-derived functional markers of inflammatory activity in Graves' disease. (A) Example pseudo color-coded MSOT images of $\mathrm{HbR}, \mathrm{HbT}$, and fat of Graves' disease and healthy thyroid tissue. Images show higher $\mathrm{HbR}$ and $\mathrm{HbT}$ and lower fat content in Graves' disease than in healthy tissue. ROls transferred from corresponding ultrasound images are shown to localize investigated thyroid lobe. (B) Quantification of MSOT for $\mathrm{HbR}, \mathrm{HbO}_{2}, \mathrm{HbT}, \mathrm{sO}_{2}$, fat, and water for thyroid lobes affected by Graves' disease and healthy thyroid tissue. Single thyroid lobes are represented by single points, with additional boxes indicating $\pm 1 \mathrm{SD}$ and additional lines representing respective means. Graves' disease shows significantly elevated $\mathrm{HbR}$ and $\mathrm{HbT}$ and significantly reduced fat compared with healthy thyroid tissue. ${ }^{*} P<0.05 .{ }^{* \star} P<0.01$.

\section{DISCUSSION}

Routine imaging of thyroid disorders includes ultrasound and, if necessary, further characterization with ${ }^{99 \mathrm{~m}} \mathrm{Tc}$-pertechnetate scintigraphy. Graves' disease can be diagnosed by clinical evaluation such as laboratory testing and ultrasound imaging, especially Doppler perfusion imaging. The quantification of tissue parameters, including $\mathrm{HbR}, \mathrm{HbO}_{2}$, the related parameters $\mathrm{HbT}$ and $\mathrm{sO}_{2}$, and fat and water content, using MSOT could provide additional important biomarkers for initial evaluation, differential diagnosis, and therapy monitoring.

Higher $\mathrm{HbR}$ and $\mathrm{HbT}$ and lower fat content in Graves' disease tissue than in healthy thyroid tissue as observed in our study are consistent with, although not specific for, the pathophysiology of an antibody-mediated inflammation of the thyroid with variable multifocal lymphocytic infiltrates (23). Graves' disease-related hyperperfusion, a result of increased hormone production and stimulation of the thyroid and proven by Doppler imaging 
(24), is reflected by significantly higher HbT in our study. In contrast to Doppler imaging, MSOT additionally allows the semiquantitative analysis of different tissue parameters that are only partly related to blood flow, such as $\mathrm{sO}_{2}$ and $\mathrm{HbT}$. Significantly higher HbR for Graves' disease tissue than for healthy tissue, as seen in our results, consecutively underlines the fact that the antibody-activated (TRAK) thyroid gland needs oxygen. The highly variable tissue remodeling process obviously depends on the current status of ongoing inflammation (TRAK, ultrasound/Doppler) and administration of antithyroidal drugs $(2,23)$. In Graves' disease, the thyroid is characterized by follicular hyperplasia and reduction of follicular colloid, which can potentially explain the significantly lower fat content than in healthy tissue in our study. By assessing such functional tissue markers, MSOT could establish a noninvasive insight into ongoing inflammation and tissue remodeling of the thyroid gland in Graves' disease not possible with ultrasound/Doppler imaging. Together with state-ofthe-art imaging (ultrasound/Doppler; scintigraphy), laboratory testing, and clinical evaluation, additional assessment of functional tissue markers by MSOT could support initial evaluation and therapy monitoring in Graves' disease patients.

Thyroid nodules are common, and their pathophysiologic differentiation (benign/malignant) remains challenging while differentiated thyroid cancer becomes increasingly prevalent (5). Following international guidelines, thyroid nodules can be noninvasively graded on the basis of ultrasound patterns $(5,9,10)$. The use of Doppler imaging is not routinely recommended for risk stratification of thyroid nodules $(6,7)$; however, it can help to distinguish between different tissues and to detect the limits of a nodule. New functional parameters assessed by MSOT, going beyond perfusion imaging with Doppler, might help to introduce new biomarkers/risk factors for risk stratification. In line with preliminary ex vivo results (16), malignant nodules, although constituting only quite a small group $(n=3)$ in our pilot study, exhibited significantly lower $\mathrm{SO}_{2}$ than benign nodules. This lower $\mathrm{sO}_{2}$ could reflect increased oxygen consumption by malignant nodules resulting in neovascularization. The resulting small vessels were reported to be detected with higher sensitivity in optoacoustic imaging than in Doppler ultrasound $(18,19)$. The lower fat content of malignant nodules in the previous ex vivo study (16) could also be reproduced in our study in vivo, possibly reflecting high cellularity of tumor tissue compared with benign nodules. The easily feasible, additional assessment of these functional biomarkers and tissue parameters by MSOT, combined with a standard of care that includes risk stratification of thyroid nodules by ultrasound and FNA, could support clinical grading and follow-up of thyroid nodules. The value and position of MSOT in the multimodal algorithm of risk assessment of thyroid nodules (5-7) need to be defined in larger prospective studies.

The large SD of the MSOT parameters of malignant nodules for $\mathrm{HbR}, \mathrm{HbO}_{2}$, and $\mathrm{HbT}$ may be due to the low number of malignant nodules included in this pilot study, their different tumor stages, levels of aggressiveness, and tumor sizes (Table 2 ), and technical limitations. Benign nodules are a very heterogeneous group as well, ranging from restrictive adenoma with high cellularity to hyperfunctional, fast-growing nodules with cystic components.

A further limitation of this pilot study is the comparably limited number (8) of wavelengths used for data acquisition. Newest developments allow the application of significantly more (e.g., 28) wavelengths within an acceptable time window, resulting in more reliable spectral unmixing. Currently, MSOT is still prone to several artifacts originating, for example, from the limited view of the probes and from perturbations from overlying tissue such as large blood vessels producing high MSOT signal. Advances in the technology of handheld optoacoustic devices, image reconstruction, and unmixing algorithms are needed to overcome current limitations.

\section{CONCLUSION}

We present first evidence for the applicability and diagnostic potential of hybrid MSOT/ultrasound imaging in thyroid disorders. Larger prospective studies are needed to corroborate our observations.

\section{DISCLOSURE}

This study was supported in part by the IZKF Münster, project Z04 and Core Unit PIX. Wolfgang Roll was funded by a rotational clinician scientist position of the Medical Faculty, University of Münster, Germany. Niklas Markwardt has received funding from the European Union's Horizon 2020 research and innovation program under grant agreement 667933 (MIB), and Angelos Karlas is supported by the Deutsche Forschungsgemeinschaft (DFG), Sonderforschungsbereich-824 (SFB-824), subproject A1. The research leading to these results has received additional funding from the Deutsche Forschungsgemeinschaft (DFG), Germany (Gottfried Wilhelm Leibniz Prize, 2013; NT 3/10-1). Jing Claussen is an employee of iThera Medical, München. Vasilis Ntziachristos is a stakeholder of iThera Medical, München. No other potential conflict of interest relevant to this article was reported.

\section{KEY POINTS}

QUESTION: Can imaging with hybrid multispectral optoacoustic tomography (MSOT)/ultrasound improve diagnostics of thyroid disease?

PERTINENT FINDINGS: Functional tissue parameters, assessed by MSOT, were analyzed in thyroid lobes affected by Graves' disease $(n=6)$, thyroid lobes with healthy thyroid tissue $(n=8)$, and benign $(n=13)$ and malignant $(n=3)$ thyroid nodules. In Graves' disease, significantly higher values for deoxygenated hemoglobin and significantly lower values for fat were found compared with healthy controls, whereas malignant thyroid nodules showed significantly lower saturation of hemoglobin and lower fat values than benign nodules.

IMPLICATIONS FOR PATIENT CARE: Hybrid MSOT/ultrasound provides semiquantitative functional parameters and tissue characterization to potentially improve noninvasive diagnostics of thyroid disorders.

\section{REFERENCES}

1. Léger J, Carel JC. Diagnosis and management of hyperthyroidism from prenatal life to adolescence. Best Pract Res Clin Endocrinol Metab. 2018;32:373-386.

2. Ross DS, Burch HB, Cooper DS, et al. 2016 American Thyroid Association guidelines for diagnosis and management of hyperthyroidism and other causes of thyrotoxicosis. Thyroid. 2016;26:1343-1421.

3. Tan GH, Gharib H. Thyroid incidentalomas: management approaches to nonpalpable nodules discovered incidentally on thyroid imaging. Ann Intern Med. 1997;126:226-231.

4. Guth S, Theune U, Aberle J, Galach A, Bamberger CM. Very high prevalence of thyroid nodules detected by high frequency (13 MHz) ultrasound examination. Eur J Clin Invest. 2009;39:699-706.

5. Haugen BR, Alexander EK, Bible KC, et al. 2015 American Thyroid Association management guidelines for adult patients with thyroid nodules and differentiated 
thyroid cancer: the American Thyroid Association guidelines task force on thyroid nodules and differentiated thyroid cancer. Thyroid. 2016;26:1-133.

6. Tessler FN, Middleton WD, Grant EG, et al. ACR Thyroid Imaging, Reporting and Data System (TI-RADS): white paper of the ACR TI-RADS committee. J Am Coll Radiol. 2017;14:587-595.

7. Russ G, Bonnema SJ, Erdogan MF, Durante C, Ngu R, Leenhardt L. European Thyroid Association guidelines for ultrasound malignancy risk stratification of thyroid nodules in adults: the EU-TIRADS. Eur Thyroid J. 2017;6:225-237.

8. Poller DN, Baloch ZW, Fadda G, et al. Thyroid FNA: new classifications and new interpretations. Cancer Cytopathol. 2016;124:457-466.

9. Chaigneau E, Russ G, Royer B, et al. TIRADS score is of limited clinical value for risk stratification of indeterminate cytological results. Eur J Endocrinol. 2018; 179:13-20.

10. Schenke S, Zimny M. Combination of sonoelastography and TIRADS for the diagnostic assessment of thyroid nodules. Ultrasound Med Biol. 2018;44:575-583.

11. Ntziachristos V, Ripoll J, Wang LV, Weissleder R. Looking and listening to light: the evolution of whole-body photonic imaging. Nat Biotechnol. 2005;23:313-320.

12. Taruttis A, Timmermans AC, Wouters PC, Kacprowicz M, van Dam GM, Ntziachristos V. Optoacoustic imaging of human vasculature: feasibility by using a handheld probe. Radiology. 2016;281:256-263.

13. Masthoff M, Helfen A, Claussen J, et al. Use of multispectral optoacoustic tomography to diagnose vascular malformations. JAMA Dermatol. 2018;154: 1457-1462.

14. Knieling F, Neufert C, Hartmann A, et al. Multispectral optoacoustic tomography for assessment of Crohn's disease activity. N Engl J Med. 2017;376:1292-1294.
15. Becker A, Masthoff M, Claussen J, et al. Multispectral optoacoustic tomography of the human breast: characterisation of healthy tissue and malignant lesions using a hybrid ultrasound-optoacoustic approach. Eur Radiol. 2018;28:602-609.

16. Dogra VS, Chinni BK, Valluru KS, et al. Preliminary results of ex vivo multispectral photoacoustic imaging in the management of thyroid cancer. AJR. 2014; 202:W552-W558.

17. Kang J, Chung WY, Kang SW, et al. Ex vivo estimation of photoacoustic imaging for detecting thyroid microcalcifications. PLoS One. 2014;9:e113358.

18. Dima A, Ntziachristos V. In-vivo handheld optoacoustic tomography of the human thyroid. Photoacoustics. 2016;4:65-69.

19. Yang M, Zhao L, He X, et al. Photoacoustic/ultrasound dual imaging of human thyroid cancers: an initial clinical study. Biomed Opt Express. 2017;8:3449-3457.

20. Cross P, Chandra A, Giles T, et al. Guidance on the reporting of thyroid cytology specimens: January 2016. Royal College of Pathologists website. https://www. rcpath.org/uploads/assets/uploaded/9ddf3c1d-c58f-4b8c-b89b63e0704f5a50.pdf. Accessed May 21, 2019.

21. Masthoff M, Helfen A, Claussen J, et al. Multispectral optoacoustic tomography of systemic sclerosis. J Biophotonics. 2018;11:e201800155.

22. Ding L, Luís Deán-Ben X, Lutzweiler C, Razansky D, Ntziachristos V. Efficient non-negative constrained model-based inversion in optoacoustic tomography. Phys Med Biol. 2015;60:6733-6750.

23. McIver B, Morris JC. The pathogenesis of Graves' disease. Endocrinol Metab Clin North Am. 1998;27:73-89.

24. Ralls PW, Mayekawa DS, Lee KP, et al. Color-flow Doppler sonography in Graves disease: "thyroid inferno." AJR. 1988;150:781-784. 